\title{
CASE REPORT OF HETEROTAXY SYNDROME
}

\author{
Rakesh Kumar Shahi ${ }^{1}$, Mridul Bhushan ${ }^{2}$, Niraj Chaudhari ${ }^{3}$, Rajendra Choudhary ${ }^{4}$, P. Nigam ${ }^{5}$
}

\section{HOW TO CITE THIS ARTICLE:}

Rakesh Kumar Shahi, Mridul Bhushan, Niraj Chaudhari, Rajendra Choudhary, P. Nigam. "Case Report of Heterotaxy Syndrome". Journal of Evolution of Medical and Dental Sciences 2015; Vol. 4, Issue 18, March 02;

Page: 3201-3206, DOI: $10.14260 /$ jemds/2015/465

\begin{abstract}
Heterotaxy is defined as abnormality where the internal organs demonstrate abnormal arrangement across the left-right axis of the body. This broad term includes patient with a wide variety of very complex cardiac lesions. Patients with hetrotaxy can be stratified into the subset of asplenia syndrome and polyasplenia syndrome, or the subset of heterotaxy with isomerism of the right atrial appendages and hetrotaxy with isomerism of left atrial appendages. Malposition of internal organs is a rare condition in clinical practice. Situs ambiguous is characterized by multiple congenital anomally relevant to intra-abdominal organs and cardiovascular surgery. Situs ambiguous with asplenia is associated with severe congenital heart disease and detected early, usually in the first year of life, while situs ambiguous polyasplenia is associated with less severe or no congenital heart disease and would be detected more often later in life. In this report, we present an adolescent case of situs ambiguous anomally which was diagnosed incidentally. The patient had left sided liver, multiple splenicule and interrupted inferior vena cava (IVC). Furthermore she had a history of genu valgum which was diagnosed as rickets, and corrective surgery for this was done at the age of 11 years.
\end{abstract}

KEYWORDS: Situs, Ambiguous, Polysplenia, Heterotaxy, Rickets.

INTODUCTION: Situs anomally are rare, complex, and confusing. Situs ambiguous or heterotaxy implies a disordered organ arrangement in the chest or abdomen. This unpredictable anatomy differs from orderly arrangement of the truncal organs in either the typical anatomy (Situs solitus) or the mirror image of it (situs inversus). Abnormal intestinal rotation is commonly found in situs ambiguous. During 5-6 weeks of gestation life, intra-abdominal organs are involved I some form of rotation and any nosological factor during this period can cause incomplete or reverse rotation. Heterotaxy is an incomplete laterization disorder. It can be either present with asplenia or have multiple spleens. Association with asplenia has poor prognosis.

In this report we present a case of situs ambiguous anomaly (with polysplenia syndrome) that was diagnosed incidentally at the age of 11 years.

CASE REPORT: A 17 year old female patient was admitted to medicine department in July 2013 with complaints of fatigue and abdominal discomfort. She had a past history of bowing of legs at the age of 11 years for which she underwent a genu valgum corrective surgery. Physical examination revealed mild pallor, no lymphadenopathy, no organomegaly, wheeze on chest auscultation but other findings were unremarkable. Laboratory investigation showed elevated Alkaline Phosphatese (ALP). Chest radiograph and electrocardiography examination were normal. Abdominal ultrasonography (US) revealed hepatomegaly with prominent left lobe and normal appearance of the spleen. The texture of parenchyma was coarse granular pattern suggesting chronic parenchymal disease. Furthermore para-aortic round solid masses initially reported as lymphadenopathy were noted. 


\section{CASE REPORT}

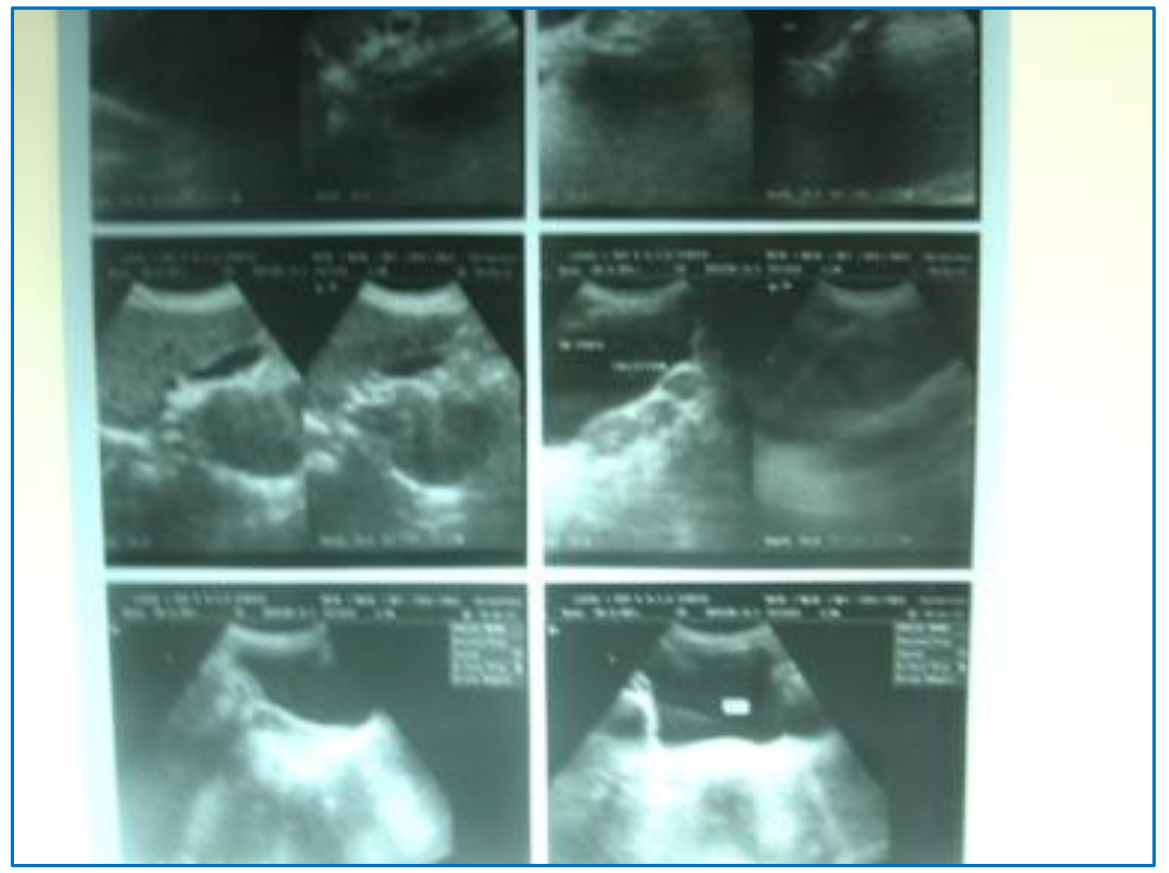

Fig. 1: US showing chronic parenchymal liver disease and intra-abdominal lymphadenopathy

In a background of high ALP and normal US we advised the patient for CT of whole abdomen but due to financial reason she couldn't afford it so we did a repeat. US this time liver was noted left side, and stomach and spleen on the right side. Gall bladder, Cystic duct and Portal vein were on left side. 2-3 hypo echoic rounded $2.2 \mathrm{cms}$. homogenous poly spleen were found. Lower part of Inferior Vena Cava (IVC) was visualized but retro hepatic part was not seen.

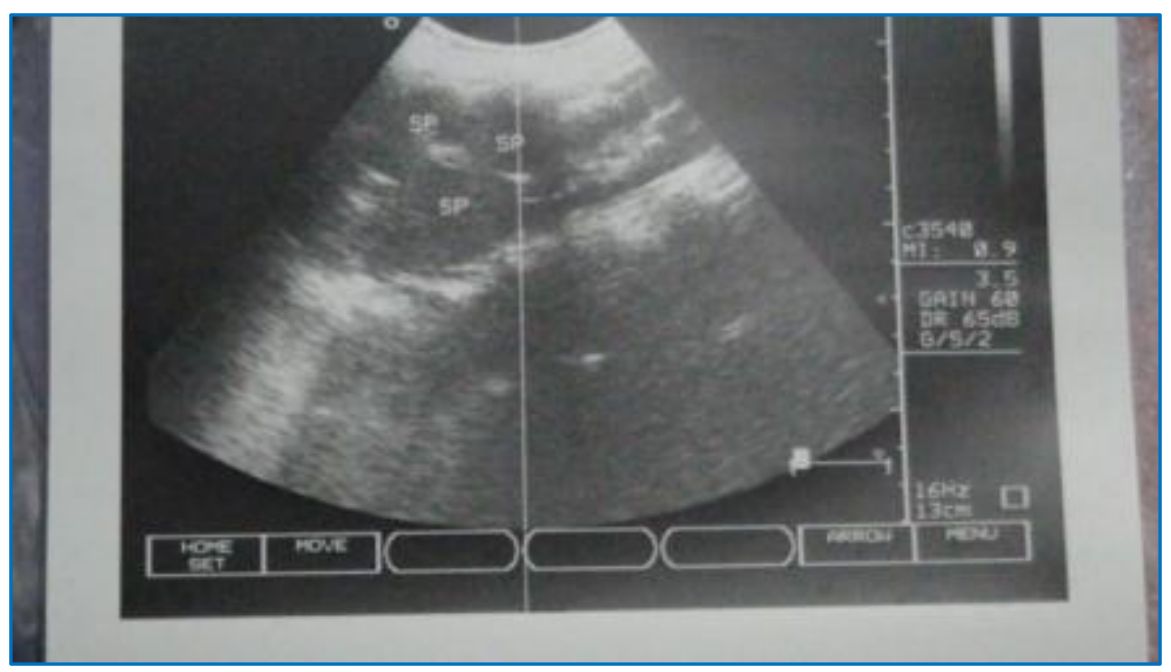

Fig. 2: Ultrasound plate showing multiple spleen and left sided liver 


\section{CASE REPORT}

Thus left sided liver with poly spleen and interruption of retro hepatic part of IVC are associated with Hetroxy syndrome, and multiple para aortic solid mass demonstrated on ultrasonography were thought to represent multiple spleen. To visualize the retro hepatic part of IVC, IVC venography was done which confirmed its absence.

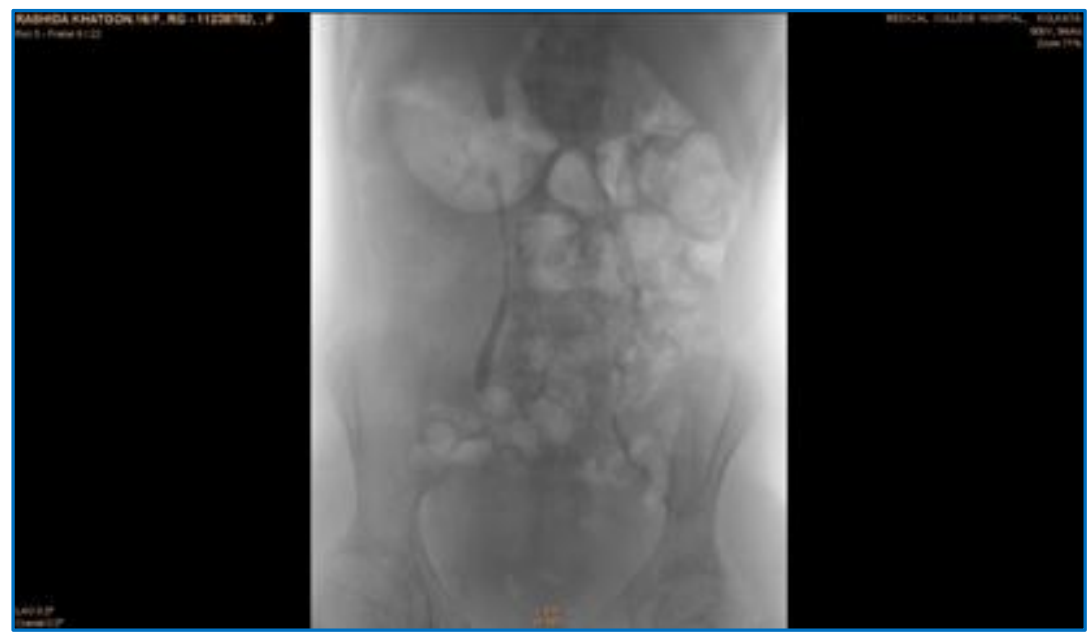

Fig. 3: Venography showing non visualization of The retro-hepatic part of IVC

Echocardiography was done to determine other anomalies related to this syndrome. It revealed a normal study (Figure 4).

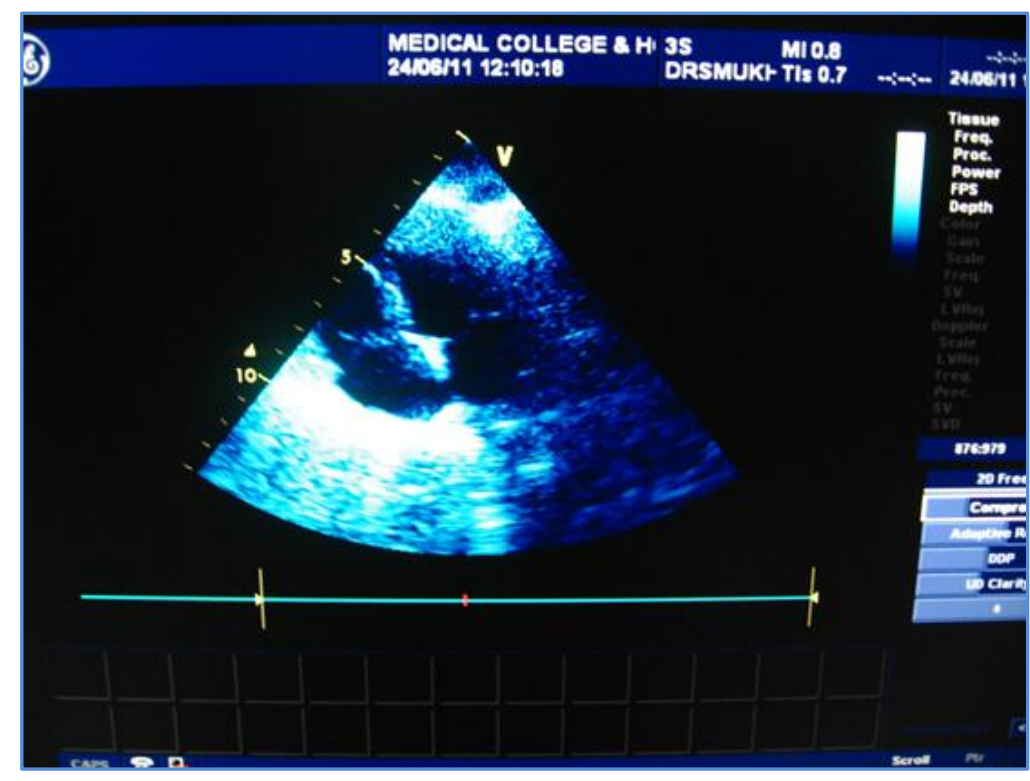

Fig. 4: Normal Echocardiographic study 


\section{CASE REPORT}

Hand and foot deformity of the patient was not relevant to polysplenia syndrome (figure5, 6). It could be explained by nutritional deficiency which is common in low socio economic strata of this part of Indian subcontinent.

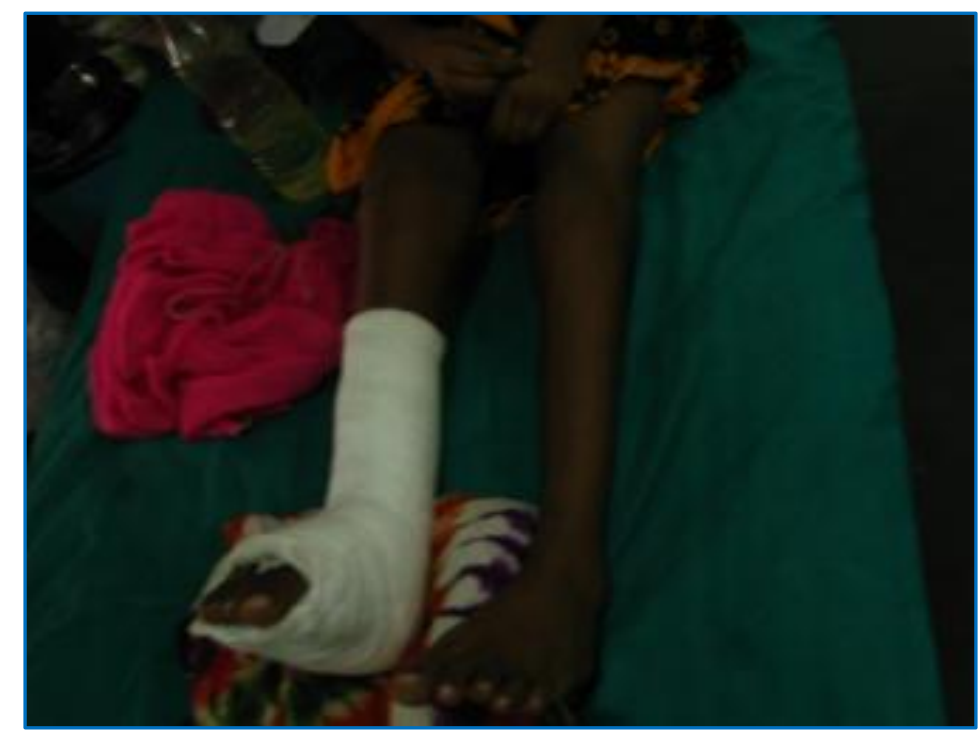

Fig. 5: Lower limb deformity of the Patient.

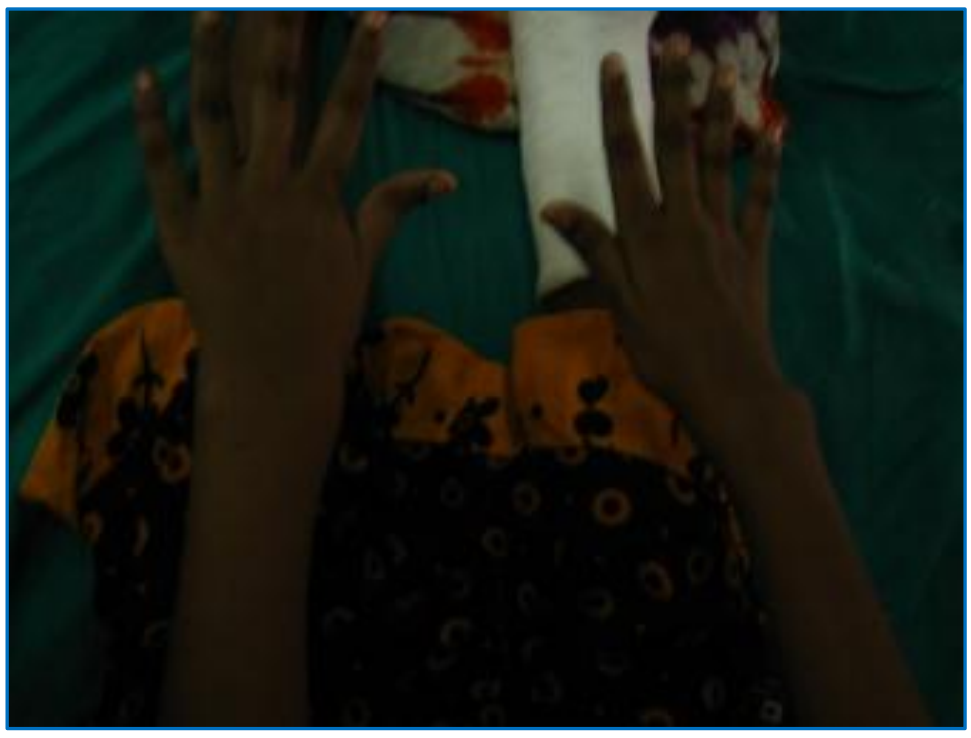

Fig. 6: Hand deformity of the patient

We did wrist joint X ray (figure7) and serum calcium $(9.2 \mathrm{mg} / \mathrm{dl})$, serum phosphate $(2.40$ $\mathrm{mg} / \mathrm{dl}$ ), and serum PTH (44.20), $25(\mathrm{OH})$ Vitamin D (14.30). Patient was diagnosed as a case of Rickets and treated with Vitamin D supplementation. 


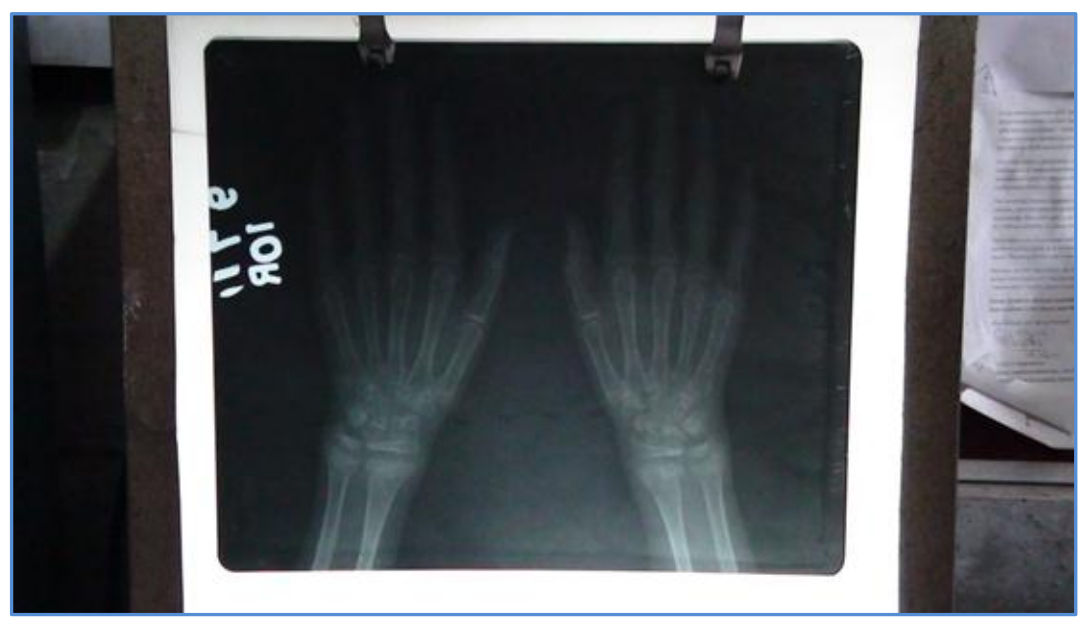

Fig. 7: X - Ray hand (PA view)

DISCUSSION: Situs ambiguous or heterotaxy syndrome refers to malposition and dysmorphism of thoracic and atrial arrangement and vascular anomalies. Azygous and hemiazygos constitution of IVC with absence of the hepatioc segment is the most frequent associated anomaly. Primitive streak appears in third week of gestation caudally to embryonic disc. It is important structure for identification of right and left side. First asymmetry appears in fourth week when heart tube loops to right. ${ }^{1}$ In sinus solitus the viscera are in normal position. When the abdominal viscera are in mirror image they are known as situs inversus.

If site of viscera cannot be readily determined it is termed as situs ambiguous or situs indeterminus. The incidence of situs ambiguous is 0.25 in $10000 .^{2,3} \mathrm{~A}$ typical presentation of this syndrome can be known by the fact that Situs ambiguous has two variants, asplenic syndrome and polysplenic syndrome. Absence of hepatic segment of IVC is most frequent congenital abnormality. ${ }^{4}$ Moreover this syndrome includes wide variety of clinical manifestation, and no single anomaly is pathognomonic. Most of the cases are sproradic but familial cases have been reported. Mortality and morbidity mainly depends on the degree of congenital heart disease.

Our patient presented with non-specific symptoms. Liver parenchymal disease was excluded by liver biopsy. The most likely reason that our patient with heterotaxy syndrome was asymptomatic and felt well until she was 16 years of age was that she had no relevant structural or functional cardiac defect. 5 Overall, the presentation of heterotaxy syndrome in adulthood as an incidental finding is rare. ${ }^{6}$ There are a broad variety of clinical manifestations in heterotaxy syndrome, which can present late in adulthood if substantial cardiovascular defects are absent.

The number of asymptomatic patients with heterotaxy syndrome diagnosed in adulthood may rise with the increasing utilization of $\mathrm{CT}$ and magnetic resonance imaging ${ }^{7}$. In summary, situs ambiguous anomaly is an embryologically and anatomically well-known condition. It is mainly encountered in Pediatric age group but it should be kept in mind when abnormal findings are encountered in advanced age group. 


\section{REFERENCES:}

1. Moore KL, Persaud TVN. Formation of germ layers and early tissue and organ differentiation: the third week. In: Moore KL, ed. The Developing Human: Clinically Oriented Embryology. 6th ed. Philadelphia: Saunders, 1998; 63-82.

2. Rose V, Izukawa T, Moes CAF. Syndromes of asplenia and polysplenia: a review of cardiac and non-cardiac malformations in 60 cases with special reference to diagnosis and prognosis. $\mathrm{Br}$ Heart J 1975; 37: 840-52.

3. Van Praagh R, Weinberg PM, Smith SD, et al. Malpositions of the heart. In: Adams FH, Emmanouilides GC, Riemenschneider TA, eds. Moss' Heart Disease in Infants, Children, and Adolescents. 4th ed. Baltimore: Williams \&Wilkins, 1989; 530-80.

4. Bernstein D. Cyanotic congenital heart disease: lesions associated with increased pulmonary blood flow. In: Berhman RE, Kliegman RM, Jenson HB, eds. Nelson Textbook of Pediatrics. 16th ed, Vol. 2. Philadelphia: Saunders, 2000; 1395-405.

5. Winer-Muram HT. Adult presentation of heterotaxic syndromes and related complexes. J Thorac Imaging 1995; 10: 43-57.

6. Fulcher AS, Turner MA. Abdominal manifestations of situs anomalies in adults. Radiographics 2002; 22: 1439-56.

7. Peoples WM, Moller JH, Edwards JE. Polysplenia: a review of 146 cases. Pediatr Cardiol 1983; 4: 129-37.

\section{AUTHORS: \\ 1. Rakesh Kumar Shahi \\ 2. Mridul Bhushan \\ 3. Niraj Chaudhari \\ 4. Rajendra Choudhary \\ 5. P. Nigam}

\section{PARTICULARS OF CONTRIBUTORS:}

1. Associate Professor, Department of General Medicine, B.R.D Medical College, Gorakhpur.

2. Assistant Professor, Department of General Medicine, Government Medical College, Kannouj, U.P.

3. Senior Resident, Department of General Medicine, B.R.D Medical College, Gorakhpur.

FINANCIAL OR OTHER COMPETING INTERESTS: None
4. Associate Professor, Department of General Medicine, B.R.D Medical College, Gorakhpur.

5. Retd. Professor \& HOD, Department of General Medicine, B.R.D Medical College, Gorakhpur.

\section{NAME ADDRESS EMAIL ID OF THE CORRESPONDING AUTHOR:}

Dr. Rakesh Kumar Shahi, I-83 Rapti Nagar, $4^{\text {th }}$ Phase, Near BPCI, Gorakhpur, Uttar Pradesh- 273001.

E-mail: drrkshahigkp@gmail.com

Date of Submission: 02/02/2015. Date of Peer Review: 03/02/2015. Date of Acceptance: 17/02/2015. Date of Publishing: 02/03/2015. 